\title{
Surgimiento, evolución y dificultades del diagnóstico de transexualismo.
}

Emergence, evolution and difficulties of the transsexualism diagnosis.

\author{
María Fernández Rodríguez a , Elena García-Vega ${ }^{\text {b }}$
}

${ }^{a}$ Psicóloga Clínica. Unidad de trastornos de Identidad de Género del Principado de Asturias (U.T.I.G.P.A.), España. Hospital San Agustín de Avilés y Centro de Salud Mental I (C.S.M. I) "La Magdalena". (Área sanitaria III), España, ${ }^{b}$ Profesora Titular de la Facultad de Psicología de la Universidad de Oviedo, España.

Correspondencia: María Fernández Rodríguez (maría.fernándezr@sespa.princast.es)

Recibido: 16/05/2011; aceptado con modificaciones: 03/10/2011

RESUMEN: Se realiza un recorrido histórico a través de las distintas revisiones de la Clasificación Internacional de Enfermedades (C.I.E.) y del Manual Diagnóstico y Estadístico de los Trastornos Mentales (D.S.M.). Se pretende analizar la aparición del diagnóstico de transexualismo y su evolución hasta llegar a las clasificaciones actualmente en vigor (C.I.E.-10) y ( D.S.M.-IV-TR). Por último, se expondrán algunas de las dificultades encontradas en el proceso de diagnóstico.

PALABRAS CLAVE: Transexualismo, Diagnóstico, Trastorno de Identidad de Género.
ABSTRACT: A historical path is made through the different reviews of the International Classification of Diseases (I.C.D.) and the Diagnostic and Statistical Manual of Mental Disorders (D.S.M.). The aim is to analyze the emergence of the transsexualism diagnosis and its evolution into the classifications currently in force (I.C.D.-10) and (D.S.M.-IV-TR). Finally, some of the difficulties found during the diagnostic process will be presented.

KEY WORDS: Transsexualism, Diagnosis, Gender identity disorder.

\section{Introducción}

Como veremos a lo largo de esta exposición, la distinción entre transexualismo, transvestismo y homosexualidad es bastante reciente. Históricamente se han tratado estos temas desde las desviaciones o aberraciones sexuales, confundiendo y superponiendo las características de unos y otros. En la actualidad, aunque se ha ido perfilando y separando la transexualidad como entidad propia, no ha conseguido desligarse del ámbito de las desviaciones y de los trastornos sexuales, dando lugar a polémicos y controvertidos debates.

La transexualidad, hasta la fecha, es considerada por las distintas disciplinas que la abordan, la medicina, la psicología, la psiquiatría o la sexología, como una enfermedad mental y es la forma de presentación más grave de los trastornos de identidad de género (1). Este rótulo queda legitimado por su inclusión en las clasificaciones diagnósticas como trastorno mental. Sin embargo, es el único trastorno mental donde el objetivo terapéutico, no es mejorar o eliminar los síntomas, sino facilitarlos a través del tratamiento hormonal y quirúrgico. 
La transexualidad o trastorno de la identidad de género es definido por las clasificaciones internacionales de enfermedades en vigor $(2,3)$, como un trastorno mental caracterizado por una identificación acusada y persistente con el otro sexo, por un malestar persistente con el propio sexo y por un sentimiento de inadecuación con el rol genérico, lo cual provoca un profundo malestar psicológico y alteraciones significativas en el área social, ocupacional o en cualquier otro aspecto importante del funcionamiento.

Los protocolos de tratamiento médico, internacionalmente aceptados para atender a estas personas incluyen la cirugía de reasignación sexual, siempre y cuando el paciente cumpla con determinados criterios de elegibilidad y disposición. El protocolo del proceso transexualizador que tiene más aceptación general entre los distintos equipos de tratamiento está basado en los estándares propuestos desde la década de los 80 por la Asociación Internacional de Disforia de Género "Harry Benjamin", que recomienda la terapia triádica (psicológica, hormonal y quirúrgica), marcando criterios específicos de elegibilidad y adicionales de disposición de obligado cumplimiento tanto para la terapia hormonal como quirúrgica $(4,5)$. En el 2007 esta asociación ha cambiado de nombre y se la conoce como Asociación Mundial de Profesionales para la salud Transgénero (WPATH) (6).

El protocolo del proceso transexualizador establece que todo el proceso de cambio se deriva de la realización de un diagnóstico de transexualismo. Un diagnóstico equivocado es un factor predictivo de arrepentimiento después del tratamiento de reasignación de sexo y de la evolución post-tratamiento (7). Por lo tanto, la evaluación diagnóstica del trastorno es el primer paso que realiza el psicólogo clínico para iniciar el proceso transexualizador dentro de la terapia triádica: psicológica, hormonal y quirúrgica.

Surgimiento del diagnóstico de transexualismo en las clasificaciones internacionales: CIE y DSM.

Desde las primeras alusiones a la transexualidad en la literatura médica (8-11) hasta su inclusión en las clasificaciones diagnósticas $(12,13)$ pasó un largo periodo de investigación y construcción de este fenómeno como entidad independiente y diferenciada de la homosexualidad o del transvestismo (Cuadro 1).

La Clasificación Internacional de Enfermedades de la Organización Mundial de la Salud (O.M.S.) viene siendo utilizada desde 1900, pero sólo incluirá trastornos mentales a partir de su quinta revisión (14), donde aparece por vez primera en la Clasificación Internacional de Enfermedades, Lesiones y Causas de muerte, una sección dedicada a las "Enfermedades del Sistema Nervioso y de los Órganos de los Sentidos". 
Cuadro 1

Surgimiento del diagnóstico del transexualismo

\begin{tabular}{|c|c|c|}
\hline CIE-6 & 1948 & $\begin{array}{l}\text { Introducen el Capítulo } \mathrm{V} \text { dedicado a los trastornos mentales. Las desviaciones } \\
\text { sexuales se incluyen en los trastornos de personalidad y los casos de transexualismo } \\
\text { se contemplarían en este apartado. }\end{array}$ \\
\hline CIE-7 & 1955 & Sin variaciones respecto a la CIE-6. \\
\hline DSM-I & 1952 & $\begin{array}{l}\text { Las desviaciones sexuales también están dentro de los trastornos de la personalidad } \\
\text { y coloca la homosexualidad al mismo nivel que las perversiones sexuales. }\end{array}$ \\
\hline CIE-8 & 1966 & $\begin{array}{l}\text { Aparece el diagnóstico de transvestismo que junto al de homosexualidad continúan } \\
\text { dentro de las desviaciones sexuales, y al mismo nivel que las parafilias. }\end{array}$ \\
\hline DSM-II & 1968 & $\begin{array}{l}\text { Los casos de transexualismo continúan contemplándose como desviaciones } \\
\text { sexuales dentro de los trastornos de orientación sexual o del transvestismo. }\end{array}$ \\
\hline CRITERIOS FEIGHNER & 1972 & $\begin{array}{l}\text { Por primera vez en una clasificación, el diagnóstico de transexualismo debuta } \\
\text { como una de las } 14 \text { categorías diagnósticas de mayor incidencia clínica. }\end{array}$ \\
\hline$R D C$ & 1975 & $\begin{array}{l}\text { No mencionan explícitamente al transexualismo, pudiéndose incluir en la categoría } \\
\text { de "Otros trastornos psiquiátricos". }\end{array}$ \\
\hline CIE-9 & 1978 & $\begin{array}{l}\text { El diagnóstico de transexualismo aparece por primera vez en una clasificación } \\
\text { internacional. Estaría ubicado al mismo nivel que las parafilias y las disfunciones } \\
\text { sexuales y todo ello dentro de las Desviaciones y Trastornos sexuales. }\end{array}$ \\
\hline DSM-III & 1980 & $\begin{array}{l}\text { Aparece el diagnóstico de transexualismo en la nomenclatura oficial de la } \\
\text { Asociación Psiquiátrica Americana. Añade un nuevo apartado, el de los Trastornos } \\
\text { de la Identidad Sexual, donde se incluye el transexualismo y los trastornos de } \\
\text { la identidad sexual en la infancia. }\end{array}$ \\
\hline
\end{tabular}

La sexta revisión (15) incorpora un capítulo específico, el capítulo V, que incluirá la clasificación de los trastornos mentales (CIE-6) titulada "Trastornos mentales, psiconeuróticos y de la personalidad". Este capítulo constaba de 10 categorías de psicosis, 9 de psiconeurosis y 7 de trastornos del carácter, la conducta y la inteligencia. Dentro de esta última categoría están los trastornos de personalidad (320 Personalidad Patológica) que a su vez incluyen las desviaciones sexuales (320.6 Desviación Sexual).

La Organización Mundial de la Salud ha coordinado y aprobado en asambleas generales las posteriores revisiones de la Clasificación Internacional de Enfermedades que fueron puestas en vigor en los años, 1955 (CIE-7) (16), 1968 (CIE-8) (17), 1978 (CIE-9) (12) y en 1992, la décima revisión, actualmente en vigor (2).

La CIE-7 (16) apenas introdujo variaciones con respecto a la anterior edición.

En Estados Unidos el Servicio de Salud Pública organizó en 1951 un grupo de trabajo con representación de la Asociación Psiquiátrica Americana, para desarrollar una alternativa a la sección de los trastornos mentales de la CIE-6. El 
resultado de este documento será la primera edición del Manual Diagnóstico y Estadístico de los Trastornos Mentales que fue publicado en 1952 (DSM-I) (18). Las desviaciones sexuales, al igual que el alcoholismo y la dependencia de drogas, estarían también incluidas en los trastornos de personalidad.

En 1966, la Organización Mundial de la Salud aprobó la CIE-8 (17) y es publicada en 1967. Coloca las desviaciones sexuales (302) al mismo nivel que las neurosis, los trastornos de personalidad, el alcoholismo o la dependencia de drogas. Las desviaciones sexuales incluían la homosexualidad y el transvestismo y dentro de este mismo rango, la pedofilia o el fetichismo (parafilias).

La Asociación Psiquiátrica Americana en 1965 asignó a su Comité de nomenclatura y estadística la tarea de preparar un nuevo manual diagnóstico de los trastornos mentales (DSM-II) (19) basado en la clasificación de la CIE-8.

El DSM-II divide los trastornos en 10 categorías, la quinta "Trastornos de personalidad y otros trastornos mentales no psicóticos", incluye cuatro apartados diferentes: Trastornos de la Personalidad, desviaciones Sexuales, alcoholismo y dependencia de las drogas. Aunque los trastornos individuales como la pedofilia, el fetichismo, el transvestismo o la homosexualidad, no están definidos, realiza una definición introductoria de las desviaciones sexuales: "Esta categoría se reserva a los individuos cuyos intereses sexuales se dirigen primariamente hacia objetos sexuales distintos a las personas del sexo opuesto, hacia actos sexuales no asociados habitualmente al coito o hacia el coito llevado a cabo bajo circunstancias extravagantes, como puede ser el caso de la necrofilia, la pedofilia, el sadismo sexual y el fetichismo. Aunque para algunos tales prácticas no son placenteras, se ven incapaces de sustituirlas por una conducta sexual normal".

Hasta estos momentos los trastornos de la orientación, los trastornos de la identidad y las parafílias estarían en el mismo saco y los casos de transexualismo, se contemplarían como desviaciones sexuales dentro de los trastornos de orientación sexual o del transvestismo.

En el terreno de las clasificaciones diagnósticas, la aparición más importante de la transexualidad se da en los Criterios Feighner (20) convirtiéndose en base del DSM-III (13). Los Criterios Feighner fueron el primer documento elaborado por autores del movimiento neo-kraepeliano con propósitos de clasificación operativa de los 14 trastornos de mayor frecuencia clínica y fiabilidad estadística, pasando a ser un sistema taxonómico basado en definiciones precisas de las categorías diagnósticas más frecuentes.

Los Criterios Feighner fueron modificados en 1975 por Spitzer, Endicott y Robins (21) en los Criterios de Investigación Diagnóstica (R.D.C.), catálogo que incluye la definición operativa de veinticinco trastornos clínicos; con respecto a los Criterios Feighner incluyeron criterios para otros trastornos, sin embargo, no con- 
ORIGINALES Y REVISIONES

templaron ningún apartado específico para la transexualidad, pudiéndose incluir en el apartado 23 ( "Otros Trastornos Psiquiátricos").

En junio de 1974, la Asociación Psiquiátrica Americana designó a Robert L. Spitzer como presidente de una Comisión sobre nomenclatura y estadística para desarrollar un nuevo manual diagnóstico que habría de ser el DSM-III (13). La clasificación tendría que ser compatible, en la medida de lo posible con la CIE-9 (12). Esta tercera edición publicada en 1980 no fue más que la extensión de los principios adoptados en los Criterios Feighner y RDC a un número mucho más amplio de entidades diagnósticas (22).

El diagnóstico de Transexualismo aparece por primera vez en la Clasificación Internacional de las Enfermedades y Problemas Sanitarios Relacionados en 1978 (CIE-9) (12) y en la nomenclatura oficial de la Asociación Psiquiátrica Americana en 1980 (DSM-III) (13).

En la CIE-9, el transexualismo se ubica en la sección correspondiente a "Desviaciones y Trastornos Sexuales", dentro de los trastornos neuróticos, trastornos de la personalidad y otros trastornos mentales no psicóticos. Al igual que en la DSMIII, todos los trastornos sexuales están agrupados conjuntamente, así mismo los divide en transexualidad y perturbaciones de la identidad psicosexual (Trastorno de la Identidad Sexual en la Infancia en el DSM-III). El transexualismo también estaría al mismo nivel que las parafilias y las disfunciones sexuales.

El Transexualismo estaría definido como: "Desviación sexual centrada en la creencia fija de que los caracteres sexuales externos no son los que corresponden a la persona. La conducta resultante se dirige ya sea hacia el cambio de los órganos sexuales por medio de operación quirúrgica o hacia el ocultamiento completo del sexo aparente adoptando el vestido y los modales del sexo opuesto". Excluye el transvestismo "desviación sexual en que se obtiene placer sexual usando vestidos del sexo opuesto". Describe lo que en las clasificaciones actuales sería el transvestimo fetichista que se incluiría dentro de las parafilias y no dentro de los trastornos de la identidad de género, donde si se recogería el transvestismo no fetichista.

Tradicionalmente los trastornos sexuales se han dividido en dos grupos, las parafilias y las disfunciones sexuales. El DSM-III añade un tercer grupo, los trastornos de la identidad de género. Todo ello dentro de la categoría de los trastornos psicosexuales. Los trastornos de la identidad de género están caracterizados por los sentimientos de malestar o incomodidad de la persona hacia su sexo anatómico o por persistentes conductas asociadas al otro sexo.

El DSM-III tiene dos categorías específicas, con sus propias pautas diagnósticas: transexualismo y trastorno de la identidad sexual en la Infancia. Para los trastornos que se dan en la edad adulta, incluye la especificación de la orientación sexual del individuo: asexual, cuando no ha tenido deseos sexuales intensos; homosexual, con predominio de excitación sexual hacia el mismo sexo; heterosexual, 
con predominio de excitación sexual hacia el sexo opuesto y la no especificada. La aplicación de estas diferencias no es tan sencilla en la práctica clínica ya que la orientación se está definiendo partiendo del sexo biológico y no del género con el que se identifica.

\section{Evolución del diagnóstico de transexualismo en las clasificaciones internaciona- les: CIE y DSM.}

Desde la inclusión del diagnóstico de transexualismo y a lo largo de las siguientes revisiones se han ido realizando pequeños cambios en su ubicación, sin haber conseguido alejarlo lo suficiente de las disfunciones sexuales y de las parafilias. (Cuadro 2).

Cuadro 2

Evolución del diagnóstico del transexualismo

\begin{tabular}{|l|l|l|}
\hline DSM-III-R & 1987 & $\begin{array}{l}\text { Distingue los Trastornos de la Identidad de Género (TIG) de los Trastornos Sexuales. Incluye } \\
\text { los TIG en la sección correspondiente a los Trastornos de inicio en la infancia, niñez o la } \\
\text { adolescencia. } \\
\text { Añade el Trastorno de la Identidad de Género en la Adolescencia o en la vida Adulta, no transexual } \\
\text { (TISAANT). } \\
\text { El diagnóstico no ha de hacerse en aquellos casos en los que el sujeto presenta esquizofrenia con } \\
\text { ideas delirantes de pertenecer al otro sexo o en los que se da hermafroditismo. }\end{array}$ \\
\hline CIE-10 & 1992 & $\begin{array}{l}\text { Los Trastornos de la Identidad de Género son un grupo independiente de los Trastornos de la } \\
\text { Inclinación Sexual y de las Disfunciones Sexuales. } \\
\text { Dentro de los Trastornos de la Identidad de Género considera: el transexualismo, el transvestismo } \\
\text { no fetichista y el Trastorno de la Identidad de Género en la Infancia. } \\
\text { El diagnóstico no debe hacerse si es un síntoma de otro trastorno mental como esquizofrenia o } \\
\text { acompañar a cualquier anomalía intersexual, genética o de los cromosomas sexuales. }\end{array}$ \\
\hline DSM-IV & $1994 \begin{array}{l}\text { Reemplaza el término Transexualismo por el Trastorno de la Identidad de Género (TIG). } \\
\text { Vuelve a agrupar los TIG con las parafilias y las disfunciones sexuales dentro de la categoría } \\
\text { "Trastornos Sexuales y de la Identidad de Género". } \\
\text { Incluye el apartado de Trastorno de la Identidad de Género no especificado para las personas } \\
\text { que no cumplen todos los criterios (por ejemplo el transvestismo no fetichista). } \\
\text { Hace alusión a la orientación de la persona con TIG atendiendo al sexo de la persona por la que } \\
\text { se siente atraído. } \\
\text { No introduce en los criterios diagnósticos que la identidad transexual no debe ser síntoma de } \\
\text { otro trastorno mental como esquizofrenia. Este tema lo desarrolla en el diagnóstico diferencial. }\end{array}$ \\
\hline
\end{tabular}


ORIGINALES Y REVISIONES

El DSM-III fue revisado en 1987 (DSM-III-R) e introduce algunas novedades. Distingue los trastornos de la identidad de género, de los trastornos sexuales. Hay un intento de alejar los trastornos de la identidad de género de las parafilias y de las disfunciones sexuales.

Ante la problemática de dónde colocar los trastornos de la identidad de género el DSM-III-R opta por incluirlos en la sección correspondiente a los trastornos de inicio en la infancia, niñez o la adolescencia. La inclusión en este apartado no está exenta de críticas ya que muchos de los trastornos de identidad de género comienzan en la infancia, pero no todos.

Con respecto al DSM-III, añade el trastorno de la identidad de género en la adolescencia o en la vida adulta, no transexual (TISAANT) que se aplica a las personas con disforia de género leve o fluctuante. Por lo tanto el DSM-III-R, incluye cuatro categorías diagnósticas dentro de los trastornos de la identidad de género que se caracterizan por la aparición de malestar intenso y persistente acerca del propio sexo, estableciéndose diferencias en función de si aparece el trastorno en la infancia o en la edad adulta y si está o no presente el transexualismo.

Como en el DSM-III, se debe especificar la historia de la orientación sexual: asexual, homosexual, heterosexual, o la no especificada y además el diagnóstico no ha de hacerse en aquellos casos en los que el sujeto presenta esquizofrenia con ideas delirantes de pertenecer al otro sexo o en los que se da el hermafroditismo (individuos que tienen simultáneamente gónadas ováricas y testiculares).

En 1992 entra en vigor la décima revisión de la Clasificación Internacional de Enfermedades (CIE-10). A diferencia de la CIE-9, los trastornos de la identidad de género son un grupo independiente de los trastornos de la inclinación sexual y de las disfunciones sexuales. Estos tres apartados a su vez están incluidos en los trastornos de la personalidad y del comportamiento del adulto (F60-F69). Dentro de los trastornos de la identidad de género considera tres categorías diagnósticas diferentes, el transexualismo, el transvestismo no fetichista y el trastorno de la identidad de género en la infancia.

En 1994 la cuarta edición del Manual Diagnóstico y Estadístico de los Trastornos Mentales (DSM-IV) introduce cambios importantes. Mientras que la CIE10 mantiene el término transexualismo y define tres trastornos diferentes, el DSMIV, reemplaza el nombre de transexualismo por el de trastorno de la identidad de género (F64.x) y recoge en esta única categoría, los tres trastornos diferentes de la CIE-10.

Debido a lo controvertido del DSM-III-R de incluir los trastornos de la identidad de género entre los trastornos de inicio en la infancia y adolescencia, el DSMIV vuelve a agrupar estos trastornos con las parafilias y las disfunciones sexuales en una única categoría que pasa a denominarse "Trastornos Sexuales y de la Identidad de Género". 
En cuanto a las categorías diagnósticas consideradas dentro de los trastornos de la identidad de género, el DSM-IV elimina la diferenciación en función de la presencia o no de transexualismo, manteniendo únicamente una diferenciación en función de la edad del individuo.

Para las personas que no cumplen todos los criterios diagnósticos se incluye el apartado de trastorno de la identidad género no especificado En esta categoría se incluyen las personas que sólo deseaban la castración y una penectomía sin deseo de adquirir caracteres sexuales del otro sexo, aquellas con una enfermedad intersexual y disforia sexual acompañante o el transvestismo no fetichista. Este último trastorno en la CIE-10 tiene entidad propia, mientras que en el DSM-IV está dentro de los no especificados.

$\mathrm{Al}$ igual que la CIE-10, introduce entre sus criterios diagnósticos que la alteración no debe coexistir con una enfermedad intersexual. En lo referente a la orientación sexual, el DSM-IV introduce otra importante modificación con respecto al DSM-III y DSM-III-R. Las personas transexuales no se consideran homosexuales cuando se sienten atraídas por personas del mismo sexo biológico, sino todo lo contrario, partiendo del género con el que se identifican, se autodefinen como heterosexuales. El DSM-IV evita los rótulos usuales y hace alusión a la orientación de la persona con trastorno de la identidad de género atendiendo al sexo de la persona por la que se sienten atraídos. Consideran cuatro opciones para los individuos sexualmente maduros y añaden el subtipo bisexual: con atracción sexual por los hombres, con atracción sexual por las mujeres, con atracción sexual por ambos sexos y sin atracción sexual por ninguno.

En nuestra experiencia clínica hemos observado cómo podemos herir sensibilidades a la hora de hacer alusión a las anteriores clasificaciones. La persona transexual, por lo general, tiene muy clara la diferencia entre orientación sexual e identidad de género. Suelen definir su orientación desde el género sentido y desde ahí, el considerarlos como homosexuales les puede resultar hasta ofensivo. La mayoría de las transexuales femeninas manifiestan atracción por hombres heterosexuales, y del mismo modo, la mayoría de los transexuales masculinos se sienten atraídos por mujeres heterosexuales $(26,27)$.

Y para finalizar con el DSM-IV, observamos que mientras la CIE-10 establece como pauta para el diagnóstico que la identidad transexual no debe ser un síntoma de otro trastorno mental, como esquizofrenia, el DSM-IV no lo introduce en los criterios diagnósticos sino que lo desarrolla en el diagnóstico diferencial. Establece que en la Esquizofrenia rara vez hay ideas delirantes de pertenecer al otro sexo, y que la insistencia por parte del individuo de ser del otro sexo no debe ser considerada delirante, ya que lo que realmente se valora es el sentirse del otro sexo y no la creencia de pertenecer a él. En casos muy raros coexisten la esquizofrenia y el trastorno de la identidad de género. 
ORIGINALES Y REVISIONES

La última edición revisada del Manual Diagnóstico y Estadístico de los Trastornos Mentales (DSM-IV-TR) (25), aunque continúa utilizando el término de trastorno de la identidad de género, comienza a reemplazarlo por el de disforia de género. Este término es el que Money (28) recomienda en 1994 con el fin de reconquistar el campo para la psicología y la psiquiatría. Según el DSM-IV-TR los trastornos de la identidad de género se caracterizan por una identificación intensa y persistente con el otro sexo, acompañada de malestar persistente por el propio sexo. La identidad de género hace referencia a la percepción que tiene un individuo de si mismo como hombre o mujer. El término disforia de género denota sentimientos intensos y persistentes de malestar con el sexo asignado, así como el deseo de poseer el cuerpo del otro sexo y de ser considerado por los demás como un miembro del otro sexo.

La Asociación Americana de Psiquiatría presentó el 10 de febrero de 2010 el borrador de la quinta versión del Manual Diagnóstico y Estadístico de los Trastornos Mentales (DSM-V) (29) que será discutida por sus miembros y publicada en su versión final en 2013. En esta nueva versión proponen una modificación terminológica con respecto al DSM en vigor, se cambia el concepto de "Trastorno de la Identidad de Género" por el de "Incongruencia de Género" ("Gender Incongruence"). Este primer borrador no cumple las expectativas de las distintas asociaciones y grupos de personas transexuales que albergaban la esperanza de que la transexualidad desapareciera de las clasificaciones diagnósticas al igual que en su momento sucedió en los años 70 con la homosexualidad.

\section{Dificultades del diagnóstico de transexualismo desde la práctica clínica.}

Las dificultades sobre el proceso diagnóstico que a continuación vamos a desarrollar, son el resultado realizado desde la práctica y sobre todo desde la reflexión sobre esa práctica. Algunas de las dificultades van a hacer referencia al propio acto de diagnosticar mientras que otras son inherentes al propio diagnóstico nosológico.

Limitaciones del modelo médico de diagnóstico.

Una de las principales finalidades de las clasificaciones es facilitar la recogida de información que permita diagnosticar a los pacientes. El diagnóstico según el modelo de intervención de la medicina, es un paso necesario y previo al diseño de la intervención terapéutica. Una clasificación debe cumplir unos requisitos básicos que requiere el diagnóstico de un trastorno o enfermedad mental: "La pertenencia a una clase en una nosología debería, siguiendo las concepciones biologicistas, como mínimo, darnos información en relación con la etiología, la terapéutica o el pronóstico de una enfermedad" (30). Es evidente que las descripciones del diag- 
nóstico de transexualismo no proporcionan ninguna de estas informaciones. Esta extrapolación del modelo médico de enfermedad a las alteraciones psíquicas, ha sido cuestionada por numerosos autores que plantean las dificultades y limitaciones de la aplicabilidad de este modelo a la conducta (31-33). El reduccionismo de la causalidad lineal tiene que dar paso a la complejidad interactiva de los factores biológicos y socio-ambientales (34).

Si no se detecta no se puede tratar.

Debido a la trayectoria asistencial de los Servicios de Salud Mental y a la larga experiencia en trastornos mentales se supone que los psicólogos clínicos poseen cierta competencia para el diagnóstico de los trastornos emocionales y mentales (35). Sin embargo, también es bien cierto que el conocimiento y la formación sobre el tema en cuestión, trastornos de la identidad de género, es bastante desconocido y no se profundiza más allá del simple diagnóstico psiquiátrico. Según los estándares asistenciales, los profesionales de salud mental que desempeñan su labor en las unidades de trastornos de la identidad de género, deben poseer formación y experiencia clínica tanto en el diagnóstico y tratamiento de los trastornos mentales en general, como de los trastornos de la identidad de género (4-5).

Tras esta toma de conciencia hay que reflexionar sobre algo muy importante. A lo largo de nuestra trayectoria profesional apenas hemos visto personas que presentasen alguna forma de disforia de género, posiblemente el motivo no sea que no hayan estado en consulta, sino simplemente que no las hemos detectado al no estar presente en nuestros esquemas cognitivos. Posteriormente, esto se va confirmando cuando al realizar las primeras evaluaciones de las personas que han tenido contactos previos con los servicios de salud mental, manifiestan su insatisfacción con el especialista no sólo por la falta de formación y compromiso profesional, sino también por la frivolidad con que en ocasiones se trata su problema. En otros casos, y partiendo del contexto básicamente psiquiátrico en que nos movemos, existe la tendencia a psicopatologizar todas las conductas de la persona transexual haciendo una negación del problema de fondo.

Los casos más conmovedores son aquellos en que previamente a la derivación a los servicios especializados protagonizan un intenso y variado recorrido asistencial. La toma a cargo puede estar caracterizada por intervenciones de urgencia que en ocasiones provocan ingresos en la unidad de agudos. A la mala evolución, se añaden las valoraciones en clínicas de reconocido prestigio donde los distintos diagnósticos se acumulan y tratan farmacológicamente haciendo caso omiso a su trastorno de la identidad de género. Hay investigaciones que ponen de manifiesto que la detección precoz y el tratamiento integral de los trastornos de la identidad de género en la infancia y en la adolescencia, mejoran la calidad de vida, la comorbilidad psiquiátrica y la disforia de género. (5) La escucha activa y sobre todo la aceptación de la transexualidad, por parte no sólo 
ORIGINALES Y REVISIONES

del entorno más próximo, sino también del asistencial, favorece la salida de ese laberinto existencial (36).

Es una enfermedad mental pero no se trata como tal.

Las diferentes disciplinas que se ocupan de la transexualidad (la psicología, la medicina, la psiquiatría y la sexología) consideran, de forma mayoritaria, que la transexualidad es una enfermedad mental. Ya Harry Benjamin (37) en 1966 la definió como entidad patológica, y como trastorno mental se sigue clasificando en la actualidad (2-3). Desde esta perspectiva cabe hacerse el siguiente cuestionamiento "se diagnostica como enfermedad mental pero no se trata como tal". Cuando una persona declara su transexualidad, la intervención no consiste en persuadirle con métodos psicoterapéuticos o farmacológicos para que adecue el género sentido al sexo biológico. Estos tratamientos son ineficaces cuando tratan de invertir la identificación con el género opuesto (38), igual de ineficaces que si se aplican a la población general para modificar la propia identidad de género. Por el contrario, en la evaluación se trata de confirmar que este sentimiento es inalterable y persistente en el tiempo con el objeto de garantizar el éxito del tratamiento de reasignación de sexo. Por tanto, es una enfermedad mental que paradójicamente "se cura" cuando se facilitan los síntomas a través del tratamiento hormonal y quirúrgico $(4,5,39)$.

A pesar de todo, debemos reconocer que el hecho de estar incluida la transexualidad en las clasificaciones diagnósticas legitima el derecho a la asistencia sanitaria e impulsa y promueve la investigación (40). Sin embargo, no son pocos los que desde posturas menos conservadoras y atendiendo a los grandes avances sociales, defienden la desaparición (la exclusión) de esta entidad como trastorno (41), al igual que sucedió en su momento con la homosexualidad. La patologización que socialmente se hace de la transexualidad es una fuente de malestar y sufrimiento para las personas transexuales.

Deslizamiento del contexto terapéutico al judicial.

Un buen número de los primeros casos atendidos por disforia de género tienen un perfil específico Ante la escasez de recursos asistenciales que traten sus demandas, han optado por la autohormonación (42-44) y por la realización de intervenciones quirúrgicas y estéticas (feminizantes o masculinizantes), que no siempre ofrecen la necesaria calidad asistencial, poniendo en peligro su salud física y mental. Estas personas ya "experimentadas", son las primeras que cuestionan el papel de "jueces" que tienen asignado (en la jurisprudencia y en la literatura médica) los especialistas de salud mental para dar el salvoconducto para la intervención quirúrgica (45). Desde una postura "privilegiada" van relatando la información necesaria para ser considerados "verdaderos" transexuales. Reconstruyen su historia de vida y además avalan su diagnóstico a través de una experiencia de la vida real confirmada. Sienten que se cuestiona el derecho a decidir sobre su vida y que nadie mejor que ellos saben lo que sienten. Desde este deslizamiento del contexto 
terapéutico al judicial en que estamos posicionados debemos preguntarnos: ¿Con qué pruebas objetivas contamos para decidir quién es el auténtico transexual?

\section{¿Qué definiría al verdadero transexual?}

Los criterios restrictivos de Stoller (46) que definían quién es el verdadero transexual (ser afeminado, no haber pasado por etapas de masculinidad ni haberse considerado hombre, haber expresado la feminidad desde etapas tempranas, no dar valor al pene, no haber estado casado y no tener delirios) eliminaría a muchos de los que hoy día consideramos transexuales. No disponemos de pruebas objetivas, que con certeza, nos declaren quién es verdaderamente transexual (47). La construcción del diagnóstico de transexualidad se va nutriendo de su historia de vida, del deseo o la necesidad de demandas de reasignación de sexo, de la experiencia de vida real, etc. Todas ellas, por separado, no confirmarían el diagnóstico. No todas las personas transexuales hacen demanda de cirugía genital, ni todas (por diversos motivos) llevan a cabo una experiencia de la vida real completa, y no por ello dejan de ser transexuales.

Mayoritariamente las demandas de las personas transexuales hacen referencia a su deseo de adecuar el género sentido a los estereotipos de masculinidad y feminidad vigentes en la sociedad. Se sienten (hombre o mujer), pero no se ven como tal, necesitan verse para sentirse y que los demás también los vean. Todo su esfuerzo va a ir orientado a que los demás los vean como ellos se sienten. ¿Cómo medir el sentimiento de género?. Primero tendríamos que definir qué es eso de sentirse mujer u hombre. Si yo me pregunto qué soy y por qué sé que soy mujer, no sabría contestar y para dar una respuesta me iría a argumentos que tienen que ver con los roles tradicionales o con lo puramente biológico (tener pechos por ejemplo). No hay duda de que identidad de género y rol de género están conectados y como expresa Money (28) son las dos caras de una misma moneda "identidad de género es la experiencia privada del rol de género, y el rol de género es la manifestación pública de la identidad de género". Las escalas de masculinidad y feminidad, no miden sentimiento de género sino roles de género (48), pueden ser más o menos útiles para valorar qué grado de adecuación social tiene una persona respecto a los estereotipos de masculinidad y feminidad, pero resultan poco efectivas con respecto a la valoración de su sentimiento de género, es decir, de su transexualidad.

Diagnóstico diferencial: Transexualismo/Transvestismo.

La utilización de las dos clasificaciones diagnósticas internacionales, actualmente en vigor (DSM-IV-TR y CIE-10), proporcionan instrumentos necesarios e imprescindibles pero no suficientes para llevar a cabo esta compleja tarea. Sería 
ORIGINALES Y REVISIONES

muy simplista y reduccionista pensar que dentro de estos criterios está incluido todo el abanico de posibilidades. Realizar, en algunos casos, el diagnóstico diferencial con respecto a otros trastornos de la identidad de género (no transexuales) o incluso con algunas parafilias no resulta fácil ni está tan claro. Ejemplo de ello son algunos de los casos de transexuales secundarios $(49,50)$ cuya identificación con el sexo opuesto se manifiesta de forma más gradual y al inicio o en etapas intermedias de la vida adulta y cuya orientación es homosexual respecto al sexo con el que se identifican. Describen desde la infancia o adolescencia conductas transvestistas con excitación sexual que justificarían su exclusión del diagnóstico de transexualismo. Estas conductas sexuales con el tiempo pueden perder o modificar su efecto excitante acercándose más a las conductas transvestistas y haciéndonos dudar o pensar en una evolución hacia un transexualismo (51). La toma de esta decisión, cuando además no presenta ambivalencia ante la cirugía de reasignación de sexo, resulta difícil y controvertida por las evidencias de remisión espontánea (52) y el aumento del riesgo de arrepentimiento tras la cirugía (53-54).

Disforia de Género y deseo de reasignación de sexo.

El término "Disforia de Género", concepto más amplio que el de transexualismo, introducido por Fisk (55) y posteriormente utilizado en el DSM-IV-TR (3), abre la posibilidad de que otros trastornos de la identidad de género que no cumplan criterios de transexualidad puedan manifestar distintos grados de disforia de género; además también podría darse el caso contrario, que una persona transexual no presentase disforia de género, es decir que ese malestar entre género sentido y el sexo biológico hubiese encontrado alguna forma adaptativa de existir en el mundo, entonces ¿dejaría por ello de ser transexual?. Sin disforia también podría hacerse innecesaria la cirugía de reasignación de sexo, y el "deseo" de parecerse al sexo opuesto mediante intervenciones endocrinológicas y quirúrgicas ha sido desde los comienzos un criterio diagnóstico (2-3,12-13) también reflejado en las definiciones que los distintos autores han realizado sobre el fenómeno del transexualismo (37, 56-59). Lo que sí es bien cierto, hoy por hoy, en este contexto social, es que muchas personas transexuales hacen demanda no sólo de tratamiento hormonal sino también de cirugía. Esta demanda, más que un "deseo" es una necesidad porque "sienten" pero no se "ven" y necesitan verse para sentirse y que los demás también los vean.

Confusión identitaria: ¿Qué soy?

La formación de la identidad de género es un proceso complejo. No todas las personas llegan a las unidades especializadas con una identidad de género bien definida. El proceso de diagnóstico, en estos casos, va paralelo a un acompañamiento psicoterapéutico a través del cual va definiendo su propia identidad. Estos casos son vistos sobre todo en las personas más jóvenes que entran en contradicción al confrontar lo que sienten y lo que la sociedad espera de ellos. Dudas y ambivalencia los invaden haciéndoles cuestionar su propia autopercepción. 
Casos extremos son aquellos donde la sociedad, con sus roles normativos y sus expectativas ha ejercido toda su influencia sobre la persona haciéndola entrar en una gran confusión identitaria. A lo largo de la evaluación hacen explícita la gran confusión respecto a su identidad. En estos momentos de su ciclo vital dicen no saber lo que son: "no me siento ni hombre ni mujer", "cuando me miro al espejo no me veo ni una cosa ni otra", ¿qué soy?" (60).

La evaluación debe ir más allá del simple diagnóstico. No es suficiente hacer un buen diagnóstico para garantizar un buen pronóstico.

Es necesario hacer una profunda reflexión acerca de los conocimientos de los que disponemos en la actualidad sobre el fenómeno del transexualismo. Por un lado los avances tecnológicos permiten cambios sorprendentes en la apariencia humana y esta modelación del cuerpo a través de las intervenciones quirúrgicas y los tratamientos hormonales pueden proporcionar la ilusión de poder y control sobre el cuerpo. Por otro lado, los criterios que permiten llevar a cabo estas transformaciones se basan en la subjetividad del individuo. Estos tratamientos médico-quirúrgicos se caracterizan por su dureza e irreversibilidad (61) y por ello, es imprescindible promocionar la investigación que nos permita avanzar más en el adecuado diagnóstico. Mejorar los procedimientos de evaluación, no solamente favorece la tarea del clínico en la toma de decisiones, sino que además y sobre todo debe aportar a la persona transexual un mayor conocimiento sobre lo que le está pasando, disminuyendo su incertidumbre y posibilitándole estrategias de actuación alternativas.

Desde la experiencia entendemos que el diagnóstico de transexualismo va más allá de las simples clasificaciones diagnósticas. Estamos atrapados en un pensamiento dicotómico al que le hemos otorgado la categoría de "normalidad" y/o "naturalidad": sólo hay dos sexos, varones y mujeres biológicas y cada uno de ellos con unas características morfológicas y psicológicas específicas. El conocimiento y la formación de esta doble realidad, sexo y género, qué es y cómo se construye la identidad de género o la forma de entender los constructos de masculinidad y feminidad van a ser fundamentales para detectar y por lo tanto diagnosticar los trastornos de la identidad de género. 
BIBLIOGRAFÍA:

(1) Chiland C. Cambios de sexo. Madrid: Biblioteca Nueva; 1999.

(2) World Health Organization. International classification of diseases. $10^{\mathrm{a}}$ ed. Ginebra: WHO; 1992. (trad. cast.: Madrid: Meditor, 1992).

(3) American Psychiatric Association. Diagnostic and statistical manual of mental disorders. $4^{\mathrm{a}}$ ed. rev. Washington, DC: APA; 2002. (trad. cast.: Barcelona: Masson, 2002).

(4) The Harry Benjamin International Gender Dysphoria Association. The standards of care for gender identity disorders. $5^{\text {a }}$ version. Mineapolis: HBIGDA; 1998.

(5) The Harry Benjamin International Gender Dysphoria Association. The standards of care for gender identity disorders. $6^{\mathrm{a}}$ version. Mineapolis: HBIGDA, 2001.

(6) World Professional Association for Transgender Health (2007) WPATH XX. Bienal Symposium. Chicago. Recuperado de: http://blog.yam.com/readingintheruins/article/11656759

(7) Meyer W, Bockiting W, Cohen-Kettenis P, Coleman E, Diceglie D, Devor H et al. The standards of care for gender identity disorders. 6th version. Int J of Transgenderism. 2001; 5 (1).

(8) Friedreich J, 1830. Citado en: Pauly IB. Male psychosexual inversion: transsexualism. Arch Gen Psychiatry. 1965; 13: 172- 81.

(9) Esquirol E, Hunt EK. Mental maladies. A treatise on insanity. Filadelfia: Lea and Blanchard, 1845 .

(10) Frankel H. Homo mollis. Medizinische Zeitung. 1853; 22: 102-3.

(11) Westphal C. Die conträre Sexualempfindung. Arch. Psychiat. Nervenkr. 1869; 2: 73-108.

(12) World Health Organization. International classification of diseases. $9^{\mathrm{a}}$ ed. Ginebra: WHO; 1978. (trad. cast.: Madrid: Meditor, 1978).

(13) American Psychiatric Association. Diagnostic and statistical manual of mental disorders. $3^{\text {a }}$ ed. Washington, DC: APA; 1980. (trad. cast.: Barcelona: Masson, 1984).

(14) World Health Organization. International list of causes of death. $5^{\mathrm{a}}$ ed. Ginebra: WHO; 1938.

(15) World Health Organization. Manual of the International statistical classification of disease, injuries and causes of death. $6^{\mathrm{a}}$ ed. Ginebra: WHO; 1948.

(16) World Health Organization. Manual of the international statistical classification of diseases, injuries and causes of death. $7^{\mathrm{a}}$ ed. Ginebra: WHO; 1955.

(17) World Health Organization. International classification of diseases, injuries and causes of death. $8^{a}$ ed. Ginebra: WHO; 1967.

(18) American Psychiatric Association. Diagnostic and statistical manual of mental disorders. $1^{\mathrm{a}}$ ed. Washington, DC: APA; 1952.

(19) American Psychiatric Association. Diagnostic and statistical manual of mental disorders. $2^{\mathrm{a}}$ ed. Washington, DC: APA; 1968.

(20) Feighner JP, Robins E, Guze S, Woodruff RA, Winokur G, Muñoz R. Diagnostic Criteria for use in psychiatric research. Arch Gen Psychiatry. 1972; 26: 57-63.

(21) Spitzer RL, Endicott J, Robins E. Clinical criteria for psychiatric diagnosis and DSM-III. Am J Psychiatry. 1975; 132: 1187- 92.

(22) Lemos S. Clasificación y diagnóstico en psicopatología. En: Belloch A, Sandín A, Ramos F (eds.). Manual de psicopatología, I. Madrid: McGraw-Hill; 1995; p. 128-57. 
(23) American Psychiatric Association. Diagnostic and statistical manual of mental disorders. $3^{\text {a }}$ ed. rev. Washington, DC: APA; 1987. (trad. cast.: Barcelona: Masson, 1988).

(24) American Psychiatric Association. Diagnostic and statistical manual of mental disorders. $4^{\mathrm{a}}$ ed. Washington, DC: APA; 1994. (trad. cast.: Barcelona: Masson, 1998).

(25) American Psychiatric Association . Diagnostic and statistical manual of mental disorders. $4^{\mathrm{a}}$ ed. rev. Washington, DC: APA; 2000. (trad. cast.: Barcelona: Masson, 2002).

(26) Bergero T, Cano G, Esteva I, Giraldo F, Gornemann I, Álvarez Ortega P. Evaluación diagnóstica y seguimiento psicológico en la Unidad de Trastornos de Identidad de Género de Andalucía (Málaga). Cir Plast Ibero Latinoam. 2001; 27: 263- 72.

(27) Tsoi WF. Follow-up study of transsexuals after sex-reassignment surgery. Singapore Med J. 1993; 34: 515-17.

(28) Money J. The concept of gender identity disorder in childhood and adolescence alter 39 years. Sex Marital Ther. 1994; 20: 163-177.

(29) American Psychiatric Association. Diagnostic and statistical manual of mental disorders, $5^{\text {a }}$ ed. Development; 2010. Recuperado de: http://dsm5.org

(30) Guimón J. Condicionamiento del diagnóstico psiquiátrico. En J. Guimón J, Mezzich JE, Berrios GE, editores. Diagnóstico en psiquiatría. Barcelona: Salvat; 1988; p.3-8.

(31) Ezama E, Alonso Y, Fontanil Y. Pacientes, síntomas, trastornos, organicidad y psicopatología. Rev Int Psicol Ter Psicol. 2010; 10 (2): 293-314.

(32) González Pardo H, Pérez Álvarez M. La invención de trastornos mentales. ¿Escuchando al fármaco o al paciente? Madrid: Alianza Editorial; 2007.

(33) Guimón J. Limitaciones del diagnóstico según el modelo biológico. En J. Guimón J, Mezzich JE, Berrios GE, editores. Diagnóstico en psiquiatría. Barcelona: Salvat; 1988; p.195-199.

(34) Von Bertalanffy L. Teoría General de los Sistemas ( $7^{\mathrm{a}}$ ed). México: Fondo de cultura económica; 2011.

(35) García González J. La psiquiatría en la España de fin de siglo: Un estudios sobre la reforma psiquiátrica y las nuevas formas de atención en Salud mental. Madrid: Ediciones Díaz de santos, S.A; 1998.

(36) Vidal Hagemeijer A. El papel de la familia en el trastorno de la identidad sexual. En: Gómez Gil E, Esteva Antonio I. Ser transsexual. Glosa: Barcelona; 2006. p. 365-375.

(37) Benjamin H. The transsexual phenomenon. New York: Julian Press; 1966.

(38) Cohen-Kettenis PT, Gooren LJ. Transsexualism: A review of etiology, diagnosis and treatment. J Psychosom Res. 1999; 46: 315-33.

(39) Hottois G. Transexualisme. En Hottois G, Missa J.N. (eds.) Nouvelle encyclopédie de bioéthique. Bruxelles : DeBoeck Université ; 2001. p. 848-849.

(40) Polo Usaola C, Olivares Zarco D. Consideraciones en torno a la propuesta de despatologización de la transexualidad. Revista de la Asociación Española de neuropsiquiatría. 2011; 32 (110), 285302.

(41) Suess A. La despatologización trans desde una perspectiva de derechos humanos. Ponencia presentada en Jornadas Feministas Estatales. Granada, diciembre 2009. Disponible en www.feministas. org/jornadas.html.

(42) Blanchard R, Clemmensen L, Steiner BW. Heterosexual and homosexual gender dysphoria. Archives of Sexual Behabior. 1987; 16: 139-152. 


\section{ORIGINALES Y REVISIONES}

(43) Esteva I, Giraldo F, Bergero T, Cano G, Crespillo C, Ruíz de Adanas S, Rojo G, Soriguer F. Evaluación endocrinológica y tratamiento hormonal de la transexualidad en la Unidad de Trastornos de Identidad de Género de Andalucía (Málaga) Cir Plast Ibero Latinoam. 2001; 27. p.273-280.

(44) Gómez G. La atención a la transexualidad por la unidad de salud mental del Hospital Clinic de Barcelona en los últimos años. C. Med. Psicosom. 2006; 78: 55-64.

(45) Rendueles G. Egolatría. KRK. Oviedo, 2005.

(46) Stoller RJ. Sex and gender, II. Nueva York: Jason Aronson; 1975.

(47) Garaizabal C. La transgresión del género. Transexualidades, un reto apasionante. En Nieto J.A. (comp) Transexualidad, transgenerismo y cultura. Antropología, identidad y género. Madrid: Talasa Ed; 1998, p. 39-62.

(48) Bem SL. The measurement of psychological androgyny. (J Consult Clin Psychol. 1974; 42:155-162.

(49) Levine SB, Lothstein L. Transsexualism or the gender dysphoria syndromes. J Sex Marital Ther. 1981; 7: 85-113.

(50) Stoller RJ. Gender identity disorders. En: Kaplan HI, Freedman AM, Sadock BJ, editors. Comprehensive textbook of psychiatry, II. Baltimore: Williams \& Wilkins; 1980.

(51) Bancroft J. Deviant sexual behavior. Londres: Oxford University Press; 1974. (trad. cast.: Barcelona: Fontanella, 1977).

(52) Bergero T, Cano G, Esteva I, Giraldo F, Gornemann I, Álvarez Ortega P. Evaluación diagnóstica y seguimiento psicológico en la Unidad de Trastornos de Identidad de Género de Andalucía (Málaga). Cir Plast Ibero Latinoam. 2001; 27: 263-72.

(53) Gooren LJG. The biology of human psychosexual differentiation. Horm. Behav. 2006; 50: $589-601$

(54) Herman-Jeglinska A, Grabowska A, Dulko S. Masculinity feminity and transsexualism. Arch. Sex. Behav.2002; 31:527-534.

(55) Fisk NM. Gender dysphoria syndrome: the conceptualization that liberalizes indications for total gender reorientation and implies a broadly based multidimensional rehabilitative regimen. West $\mathrm{J}$ Med. 1974; 120: 386- 91 .

(56) Becerra A. Transexualidad. La búsqueda de una identidad. Madrid: Díaz de santos; 2003.

(57) Feighner JP, Robins E, Guze S, Woodruff RA, Winokur G, Muñoz R. Diagnostic Criteria for use in psychiatric research. Arch Gen Psychiatry. 1972; 26:57-63.

(58) Person E, Ovesey L. The transsexual syndrome in males. Primary transsexualism. Am J Psychoter. 1974a; 28:174-193.

(59) Stoller RJ. Sex and gender I. Nueva York. Karnak; 1968.

(60) Garaizabal C. Identidad: cuerpo, género y subjetividad. En: Becerra-Fernandez A. Transexualidad. La búsqueda de una identidad. Madrid: Díaz de Santos;2003, p. 235-244.

(61) Sociedad Española de Endocrinología y Nutrición. Guía clínica para el diagnóstico y tratamiento de los trastornos de la identidad de género. SEEN. Madrid, 2002. 